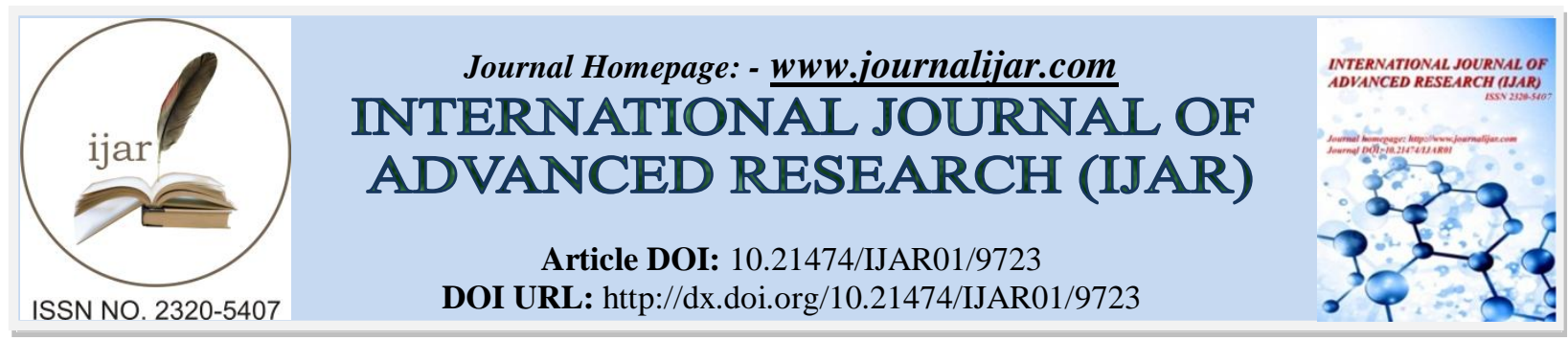

RESEARCH ARTICLE

\title{
PSYCHOLOGICAL, SOCIAL AND ECONOMIC, SATISFACTION OF DOUBLE JAW SURGERY VS. SINGLE JAW SURGERY: A RETROSPECTIVE CLINICAL STUDY.
}

Maryam Al-Hindi ${ }^{1}$, Mohammed Alkindi ${ }^{2}$, Noura AlOtaibi ${ }^{3}$, Safa Alrashed ${ }^{4}$ and Syed Sirajul Hassan ${ }^{5}$.

1. Assistant Professor and Consultant Department of Oral and Maxillofacial Surgery, Faculty of Dentistry, King Saud University.

2. Associate Professor and Chairman of Department of Oral and Maxillofacial Surgery, Faculty of Dentistry, King Saud University.

3. Lecturer, Faculty of Dentistry, King Saud University.

4. Private Sector King Saud University.

5. Assistant Consultant Oral \& Maxillofacial Surgery (Surgical Dentistry) King Saud University.

\section{Manuscript Info}

Manuscript History

Received: 09 July 2019

Final Accepted: 12 August 2019

Published: September 2019

\section{Abstract}

Orthognathic surgery involves manipulation of the jaws and skeletal structure to overcome inherited or acquired skeletal abnormalities. The psychological and social effect on these patients is recognized in many literatures. The aim of current study is to compare the quality of life in psychological, social and economic changes after orthognathic surgeries of single jaws vs. double jaws. Methods: A questioner was distributed to 40 patients in a recall visit that underwent orthognathic surgery from January 2015 to December 2017. The questioner included 27 questions divided into 7 sections out of which 3 questions related to demographic parameters such as age, gender and education level, 2 were about etiology of surgery, 3 were about orthognathic treatment plan, 12 questions were about facial changes reflecting psychological outcome of the surgery, 5 about social effect and 2 were about economical outcome of the surgery. Result: Total number of the patients in the current study was 40 out of which, 27 had a double jaw surgery while 13 had a single jaw surgery. The result was divided into four sections, including general information, physiological, social and economic section. The participant scored the highest percentage for improvement of beauty where double vs. single was 20 (64.5\%) vs. 11 (35.5\%). In regards to gummy smile improvement the participant reported $8(29.6 \%)$ vs. 4 (30.8\%) in double vs. single jaw. Lip fullness was significant in single jaw surgeries with P-value 0.015. Mandibular angle changes reported in 12 out of $27(44.4 \%)$ in double jaw comparing to 1 out of $13(7.7 \%)$ in single jaw with a significance of 0.029. In documenting post-op TMJ clicking, double jaw surgeries reported having $12(44.4 \%)$ clicking while it was only $5(38.5 \%)$ in single jaw where all patient did not experienced TMJ clicking pre surgery. Conclusion: Generally orthognathic surgery has a positive influence on the psychological, social and economic status of patients with jaw deformities. Unexpectedly there was no difference in between double and single jaw surgeries in psychological, social and economic

Corresponding Author:-Maryam Al-Hindi.

Address:-Assistant Professor and Consultant Department of Oral and Maxillofacial Surgery, Faculty of 
status except in lip fullness and mandibular angle. In the current study patient with single jaw surgeries reported increases in lip foulness while double jaw surgeries reported significant increases in the mandibular angle improvement.

Copy Right, IJAR, 2019,. All rights reserved.

\section{Introduction:-}

Orthognathic surgery involves manipulation of the jaws and skeletal structure to overcome inherited or acquired skeletal abnormalities, which could involve single or double jaws surgery. ${ }^{1}$

The main goal of those surgeries is to attain satisfaction esthetically, functionally and pyshologically. ${ }^{2}$

Generally complication of these surgeries includes bleeding, infection, temperomandibular joint symptoms and nerve injuries ${ }^{(1,3,4,5)}$. The psychological and social effect on these patients is recognized in many literture. ${ }^{6,7,8}$

Brunault et.al (2016) reported that the perception of patients for psychosocial benefits of treatment for 286 patient were positive; where $27(67.5 \%)$ patients stated that their social communication significantly enriched and $30(75 \%)$ patient's reported improvement of self-confidence. ${ }^{9}$

Corso et.al (2015) reported that there was an improvement in the quality of life after orthognathic surgery for all groups of dentofacial deformities, particularly vertical and anteroposterior deficiency of the maxilla and excess of the mandible. There was a lesser reduction of negative impact for transverse jaw deformities when compared with other deformities. ${ }^{10}$

The aim of current study is to assess the quality of life, psychological, social and economic changes after corrective surgeries of single jaw as compared to double jaw surgery.

\section{Material and methods:-}

This retrospective study was conducted in oral and maxillofacial surgery department - College of Dentistry, King Saud University, Saudia Arabia. Approval of CDRC \# IR 0286 was obtained. It included patients who underwent orthognathic surgery from January 2015 to December 2017.

Inclusion criteria involved patient who underwent orthognathic surgery for double or single jaw, above 18 years of age, no major medical illness, post-operative follow-up of at least six months. Exclusion criteria included patients younger than 18 years; medically compromised, patient with craniofacial abnormalities, cleft lip, mental retardation, psychiatric treatment and pregnant patients.

A questioner was distributed to 40 patients in a recall visit by a post graduate of oral and maxillofacial surgery department and by phone for those who couldn't come up for the follow-up visit. During the period of answering the survey, a post-graduate to clarify any unclear question accompanied patients.

The Questioner included 27 questions divided into 7 sections out of which 3 questions were, personal including age, gender and education level,

1. 2 were about causes of surgery,

2. 3 were about orthognathic treatment plan,

3. 12 questions were about facial changes reflecting psychological outcome of the surgery,

4. 5 about social effect and finally

5. 2 were about economical outcome of the surgery.

Psychological impaction section involved multiple questions about changes in the nose shape and width, gummy smile, check changes, mandibular angle, chin and lip fullness, phonetic defect, temporomandibular joint involving clicking and limitation of mouth opening and tongue movement. This section was numbered in a scale from 1 to 10 where $1(10 \%)$ indicating not satisfied and $10(100 \%)$ indicating complete satisfaction. 
All questioner papers were anonymous to maintain the patient privacy and each patient was given a number to identify the subject. The questioner papers were kept with the main researcher in his private locker with the ability of the second researcher to have access to it.

\section{Statistical Analysis:-}

PSSC version 20 was used to analyze the data. Descriptive analyses were used to evaluate the responses of participants to each section of the questionnaire. The age, gender, level of education of the subject at the study was also calculated. The characteristics of participants and non-participants in the survey were compared using chi square tests. The data were compared based on the surgeries type conducted as single jaw or double jaw, a chisquare was used with a P-value $<0.05$ to compare the outcome.

\section{Results:-}

Total number of the patients in the current study was 40 where 15 (37.5\%) were male and $25(62.5)$ were female. 16 $(40 \%)$ were between age group of nineteen and twenty-four years, $9(47.5 \%)$ were between twenty-five and thirty years and only $5(12.5 \%)$ were above thirty years.

27 patients had a double jaw surgery while 13 had a single jaw surgery.

As in regards to the level of education $6(15 \%)$ patients had high school degree, while the majority of the patient 29 $(72.5 \%)$ had a bachelors degree and only $5(12.5 \%)$ patients had a postgraduate degree.

For the second section exploring the reasons for surgery, the participant scored the highest percentage for improvement of beauty where was $20(64.5 \%)$ as compared to $11(35.5 \%)$ in double jaw and single jaw surgeries respectively. The second reason was improvement-chewing function, which account for $9(69.2 \%)$ as compared to 4 (30.8\%) in double and single jaw surgeries respectively. Third reason was improvement of the speech, which account for $8(80 \%)$ as compared to $2(20 \%)$ for double and single jaw surgeries respectively. Improvement of TMJ clicking was the $4^{\text {th }}$ cause of surgery where none reported in double jaw while it was surprisingly $3(100 \%)$ in single jaw surgery with a significance of P-value 0.009.

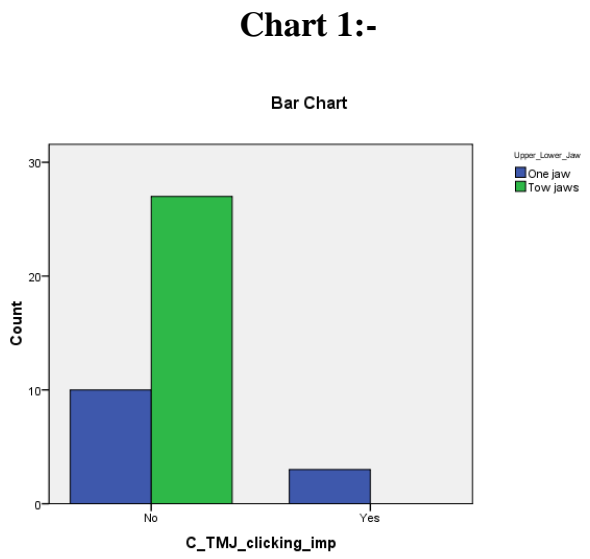

\section{Physiological effect:}

The fourth section in the study was for evaluating physiological changes occurred after surgery including gummy smile, post-operative numbness, check fullness, lip fullness, mandibular angle and chin changes, chewing, speech and TMJ clicking improvement, limitation of mouth opening and tongue movement

In regards to gummy smile improvement the participant reported 8 (29.6\%) as compared to 4 (30.8\%) in double jaw surgery and single jaw surgery respectively.

In respects to facial numbness post-operatively; the participant replied that 1 patient from each group had none while 2 patients from each group had facial numbness up to 2 weeks in both double and single jaw group. Where-as it ranged from 2-3 months for 12 participants who underwent double jaw surgery and 6 who underwent single jaw 
surgery. Numbness disappeared after 6 months for 5 patients who underwent double jaw surgery and 1 patient who underwent single jaw surgery while it was 1 patient from each group, which took 2 years to recover from numbness. Cheek fullness reported as positive in $8(61.5 \%)$ patients and $25(82.5 \%)$ with no changes in patients who underwent double jaw surgery whereas it is positive in $2(7.4 \%)$ patients and $4(30.8)$ patients with no changes in patients who underwent single jaw surgeries.

Lip fullness was reported positive in double jaw surgery vs. single jaw surgery as $1(3.7 \%)$ vs. $4(30.8 \%)$ with a significant of 0.015 (Table1)

Table 1:-Satsfaction_Lip_fulness * Upper_Lower_Jaw

\begin{tabular}{|c|c|c|c|c|c|}
\hline & & & \multicolumn{2}{|c|}{ Upper_Lower_Jaw } & \multirow[t]{2}{*}{ Total } \\
\hline & & & One jaw & Tow jaws & \\
\hline \multirow[t]{6}{*}{ Satsfaction_Lip_fulness } & \multirow[t]{3}{*}{ No } & Count & 9 & 26 & 35 \\
\hline & & $\begin{array}{l}\% \\
\text { Satsfaction_Lip_fulness }\end{array}$ & $25.7 \%$ & $74.3 \%$ & $100.0 \%$ \\
\hline & & \% within Upper_Lower_Jaw & $69.2 \%$ & $96.3 \%$ & $87.5 \%$ \\
\hline & \multirow[t]{3}{*}{ Yes } & Count & 4 & 1 & 5 \\
\hline & & $\begin{array}{l}\% \\
\text { Satsfaction_Lip_fulness }\end{array}$ & $80.0 \%$ & $20.0 \%$ & $100.0 \%$ \\
\hline & & \% within Upper_Lower_Jaw & $30.8 \%$ & $3.7 \%$ & $12.5 \%$ \\
\hline \multirow{3}{*}{\multicolumn{2}{|c|}{ Total }} & Count & 13 & 27 & 40 \\
\hline & & $\begin{array}{l}\% \\
\text { Satsfaction_Lip_fulness }\end{array}$ & $32.5 \%$ & $67.5 \%$ & $100.0 \%$ \\
\hline & & \% within Upper_Lower_Jaw & $100.0 \%$ & $100.0 \%$ & $100.0 \%$ \\
\hline
\end{tabular}

Mandibular angle changes reported in 12 out of 27 (44.4\%) in double jaw comparing to 1 out of $13(7.7 \%)$ in single jaw with a significance of 0.029 (Table 2)

Table 2:-Did_U_had_any_Mandibular_Angle_Changes? Upper vs.Lower_Jaw Crosstabulation

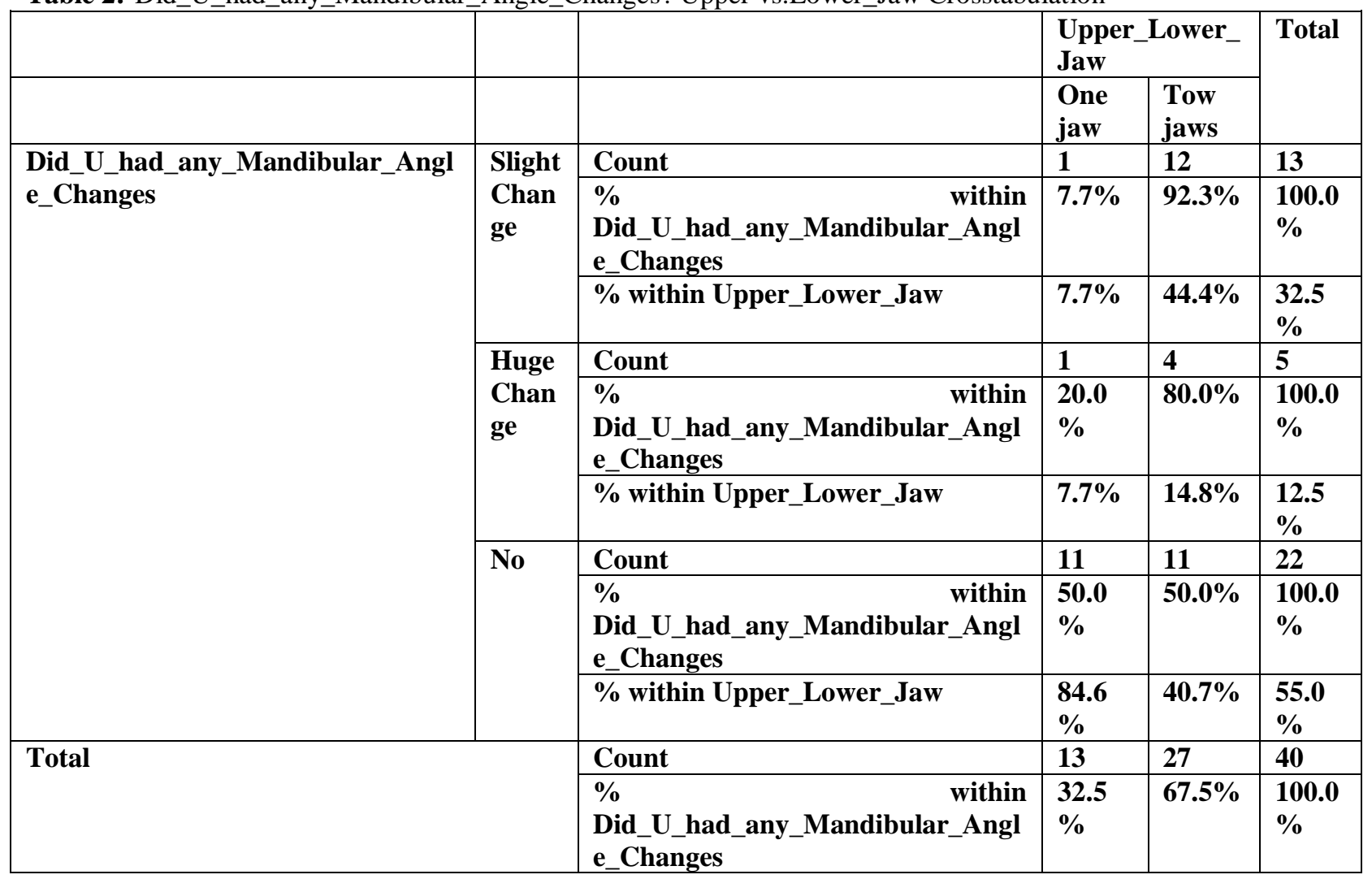


Genioplasty was performed in 11 patients where 10 were performed along with double jaw surgery while only 1 patient was performed along with single jaw surgery.

Chin changes irrespective of genioplasty reported in 20 (74.1) patients who underwent double jaw surgery and 10 (76.9\%) patients who underwent single jaw with no statistical significance.

Chewing ability improved in 11 (73.3) patients who underwent double jaw and 4 (26.7) patients who underwent single jaws.

Speech improvement was reported positive in one patients who underwent double jaw surgery comparing to neutral response in all of single jaw surgical patients with no significance.

Post-Operative TMJ clicking was present in $12(44.4 \%)$ patients who underwent double jaw surgery while it was 5 (38.5\%) patients who underwent single jaw. All subjects were free of TMJ clicking pre operatively.

In reporting limitation of mouth opening $33.3 \%$ (9 in 27) was found in patients who underwent double jaw surgery and $30.8 \%$ (4 in 13) patients who underwent single jaw surgery.

For difficulty in tongue movement post-surgery, $5(18.5 \%)$ patients who underwent double jaw surgery had tongue movement difficulty and 2 (15.4\%) patients who underwent single jaw surgery.

In the question of post-op psychiatric treatment only $2(7.4 \%)$ participants were exposed to psychiatry treatment from the double jaw surgery participant and none reported from single jaw surgery participant.

\section{Social effect:}

In evaluating post-operative social satisfaction 5 questions were given to the participants including participant satisfaction, family and friends satisfaction, possibility of doing the surgery again, reflection of the changes occurred post-surgical for friends, family and at work and recommendation of the participant to other about the surgery.

For participant satisfaction, $3(11.1 \%)$ vs. 1 (7.7\%) reported $70 \%$ satisfaction in double vs. single, While $80 \%$ satisfaction reported in $4(14.8 \%)$ vs. 1(7.7) in double vs. single jaw. $90 \%$ satisfaction was conveyed in $2(7.4 \%)$ vs. $4(30.8 \%)$ in double vs. single while $100 \%$ satisfaction was documented in $16(59.3 \%)$ vs. $6(46.2 \%)$ for double vs. single jaw with no significant between the two groups.

In the question of "Would you do the surgery again"? The participant replied as strongly agreed in $11(40.7 \%)$ vs. in $8(61.5 \%)$, agreed in $8(29.6 \%)$ vs. $2(15.4 \%)$, neutral in 1 (3.7\%) vs. none and disagreed in $4(14.8 \%)$ vs. 1 (707\%) for double vs. single jaw surgeries correspondingly.

In the question of "Would you recommend the surgery to others"? The participant answered as strongly agreed in 19 $(70.4 \%)$ vs. $12(92.3 \%)$, agreed was documented in $4(14.8 \%)$ vs. $1(7.7 \%)$, neutral in $3(11.1 \%)$ vs. none and disagreed in $1(3.7 \%)$ vs. none in double vs. single jaw surgeries correspondingly.

For the question of "Did you have difficulty for family and friends recognizing you"? The participant answered as yes in $14(51.9 \%)$ vs. $3(23.1 \%)$ and no in $13(48.1 \%)$ vs. $10(76.9 \%)$ in double vs. single with no significant between the two groups.

For the question "Did family and friends liked your face"? The participants replied in double jaw as yes in 26 (96.3\%) comparing to $13(100 \%)$ in single jaw with no significance.

For the question of "Satisfaction of family and friends towards changes in your face" ? The participants in double vs. single jaw surgeries replied as very satisfied in $20(74.1 \%)$ vs. $8(61.5 \%)$, and satisfied in $7(25.9 \%)$ vs. 4 $(30.8 \%)$ with no significance. 
For the part of admiring the participant new face by family and friends, the double jaw surgeries reported 23 (85.2\%) out of 27 comparing to $7(53.8 \%)$ out of 13 in single jaw

For the question "Did you have any improvement in your social relationship"? The participants answered in double vs. single jaw as yes in $17(63 \%)$ comparing to 6 (46.2\%) and no in $10(37 \%)$ vs. in $7(53.8 \%)$.

\section{Economic effect:}

For work relationship improvement, the participant answered range from $11(40.7 \%)$ out of 27 for double jaw to 3 $(29.1 \%)$ out of 13 in single jaw surgeries.

For financial outcome improvement, double jaw reported none compared to I (7.7\%) in single jaw.

For the question if the surgery cost them financially, double jaw surgeries reported 6 (22.2\%) out of 27 while none reported in single jaw.

\section{Discussion:-}

1. A comparison between double and single jaw surgeries in physiological, social and economic impact was not found in literature and to our knowledge this is the first comparative clinical study reported.

2. The number of the patient in the current study is in consistent with the study of Posnick and Wallace ${ }^{6}$. The increased number of the female in the current study is in consistent with many studies ${ }^{6,11}$.

3. Level of education was not significant in many reported similar studies ${ }^{6,12}$ while it were reported in the current study as the majority of the patient had a pachler degree $29(72.5 \%)$.

4. Psychological effect: In the current study, improvement of beauty reported as the highest cause of surgeries in double jaw vs. single jaw which in consistent with Meadea \& Inglehart $2010{ }^{12}$ where significant relation was reported between esthetic and satisfaction and with Rispoli and his coworkers $2004 .^{13}$

5. Improvement of TMJ was reported in the current study as $3(100 \%)$ in single jaw with a significance of P-value 0.009 where none reported in double jaw and this is in consistent with the study of Al-Riyami et al $2009^{23}$ where improvement of TMJ clicking and crepitation was noted.

6. In the current study one case from each group reported having numbnous up to 2 years post-operatively and none from the two groups reported having permanent loss of sensation. This is not in agreement with Posnick and Wallace 2008 where they reported loss of sensation in $21 \%$ ( 9 of 42 ).

7. In the current study lip fullness was reported with no significant which is in agreement with many stadies. ${ }^{2,6}$

8. In the current study check fullness was significant between double and single jaw surgeries with $P$ value $<0.015$ which not in consistent with Posnick and Wallace ${ }^{6} 2008$.

9. Change in mandibular angle in the current study is in consistent with the systematic review of Kaklamanos \& Kolokitha $2016^{24}$ where angle changes will follow the setback of the mandible.

10. Chewing ability improved in 11 (73.3) patients in double jaw vs. 4 (26.7) in single jaws which in consistent with study of Posnick and Wallace 2008 as they reported $80 \%$ occlusion improvement in their study.

11. In the current study speech improvement was reported in a single case $3.7 \%$ of double jaw which inconsistent with Posnick and Wallace 2008 where about $80 \%$ were satisfied with their speech post operatively.

12. In the current study, Limitation of mouth opening was reported in $30 \%$ of the cases which is inconsistent with the study of Tahir and others 2015 where they reported only $12.5 \%{ }^{22}$.

13. In the current study post-op TMJ clicking reported as 12 out of 27 in double jaw comparing to 5 out of 13 in single jaw surgeries, this inconsistent with the study of Al-Riyami and others $2009^{23}$ where they reported improvement in their signs and symptoms than deterioration in patients having orthognathic treatment and also suffering from TMD appear. In the systematic review of 544 article Bermell-Baviera et al 2016 found no effects of mandibular advancement surgery on the temporomandibular joint neither to improve nor to worsen TMJ health. ${ }^{24}$

14. Social effect: In the current study satisfaction of double jaw surgeries vs. single is $85 \%$ to $78 \%$ correspondingly which in accordance to the study of Posnick and Wallace ${ }^{6} 2008$ where they reported $87 \%$ satisfaction and also to some other studies ${ }^{14,15}$.

15. For the respondent reaction about their agreement to do the surgery again, they reported positive answer in $70.4 \%$ (19 out of 27) in double jaw vs. 77\% (10 out of 13) in single jaw. This in consistent with the study of Posnick and Wallace $^{6}$, while it is not in agreement with many studies. ${ }^{12,}{ }^{16-20}$ Meadea \& Inglehart $2010^{12}$ reported $80 \%$ of their participants were reelecting to have the surgery again while they recommended the surgery to others in $79 \%$. 
16. The recommendation of the participants to other to do the surgical correction was positive in $85 \%$ (23 out of 27) for double jaw surgeries and 77\% (10 out of 13) in single jaw. This is in agreement with the study of Posnick and Wallace ${ }^{6}$ where the participants respond that they will recommend the surgery to others in $93 \%$.

17. In the question whether your family recognizes you post-operatively and if they like your new face, the answer of the participant in this study was not significant between the two group which is in consistent with Posnick and Wallace. ${ }^{6}$

18. For the question whether family and friends like your new face, the participant in the current study respond as very satisfied in $20(74.1 \%)$ for double jaw while for single jaw it was $8(61.5 \%)$. This in agreement with many studies. ${ }^{2,6,8}$

19. For the question whether family and friends admiring your new face, the participant in the current study

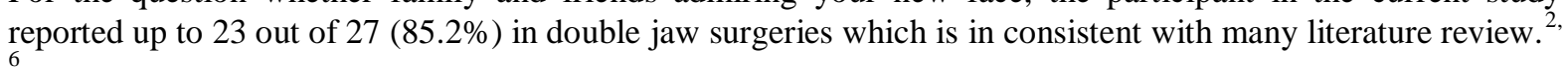

20. For the question "Did you have any improvement in your social relationship"? The participants answered were in consistent with Co-Pereiraa et al 2016. ${ }^{26}$

\section{Economic effect:}

1. For the question inquiry if the surgeries coast them, both group answered as no coast resulted from the surgeries where no such a question was asked in the literature.

2. For the question if the post surgeries increase their personal income participant reported having no effect of the surgery on their income.

\section{Conclusion:-}

1. In conclusion, generally orthognathic surgery has a positive influence on the psychological, social and economic status of patients with jaw deformities.

2. Unexpectedly there was no difference in between double and single jaw surgeries in psychological, social and economic status except in lip fullness and mandibular angle.

3. In the current study patient with single jaw surgeries reported increases in lip foulness comparing to double jaw surgeries.

4. In the current study mandibular angle changes reported significant result in double jaw surgeries comparing to single jaw

5. The limited numbers of cases in the current study affect the general result and possible increase in cases.

\section{References:-}

1. Chrcanovic BR, Toledo GL., Amaral MB, Cusodio AL. Assessment Of hematologic parameters before and after bimaxillary orthognathic surgery. Oral Maxillofac Surg Aug 2015; 20: 35-43

2. TAHIR S, RAFFI S, IBRAHIM MW, KHAN MN. Esthetics, functional, and psychosocial satisfaction after orthognathic surgery in patients having dentofacial skeletal deformities. Pakistan Oral \& Dental Journal Sep 2015; 35(3): 395-400

3. Chow LK, Singb B, Cbiu WK, Samman N. Prevalence of postoperative complications after orthognathic surgery: A 15-year review. J Oral Maxillofac Surg 2007; 65: 984-992

4. Sebastiani AM, Filho FB, Bonotto D, Kluppel LE, Rebellato NL, da Costa DJ, Scariot R. Influence of orthognathic surgery for symptom ms of temporomandibular dysfunction Feb 2016; 121(2): 119-125

5. Mcleod NMH, Bowe DC. Nerve injury wiyh orthognathic surgery. Part 2: Inferior alveolar nerve. B J Oral Maxillofac Surg Feb 2016; 54:366-371

6. Posnick JC, Wallace J. Complex orthognathic surgery: Assessment of patient satisfaction. J Oral Maxillofac Surg 2008; 66: 934-942

7. Baherimoghaddam T, Tabrizi R, Naseri N, Pouzesh A, Oshagh M, Torkan S. Assessment of the changes in quality of life of patients with class II and III deformities during and after orthodontic-surgical treatment. Int J Oral Maxillofac Surg 2016; 45: 476-485

8. Takatsuji H, Kobayashi T, Kojima T, Hasebe D, Izumi N, Saito I, Saito C. Effects of orthognathic surgery on psychological status of patients with jaw deformities. Int J Oral Maxillofac Surg 2015; 44: 1125-1130.

9. Brunault P, Battini J, Potard C, Jonas C, Zagala-Bouquillon B, Chabut A, Mercier JM, Bedhet N, Re'veille`re C, Goga D, Courtois R: Orthognathic surgery improves quality of life and depression, but not anxiety, and patients with higher preoperative depression scores improve less 2016. Int J Oral Maxillofac Surg; 45: 26-34 
10. Corso PF, Costa FA, Kluppel LE, Rebellato NL, Scariot R. Evaluation of the impact of orthognathic surgery on quality of life. Braz Oral Res (online) Mar 2015; 30: e4

11. Abrahamsson C, Henrikson T, Nilner M, Sunzel B, Bondemark L, Ekberg EC. TMD before and after correction of dentofacial deformities by orthodontic and orthognathic treatment. Int. J. Oral Maxillofac. Surg. 2013; 42: 752-758. \# 2012 International Association of Oral and Maxillofacial Surgeons

12. Meadea EA and Inglehart MR. Young patients' treatment motivation and satisfaction with orthognathic surgery outcomes: The role of "possible selves". American Journal of Orthodontics and Dentofacial Orthopedics Volume 137, Number 1 2010; 137 (1):26-34

13. Rispoli A, Acocella A, Pavone I, Tedesco A, Giacomelli E, Ortiz L, et al. Psychoemotional assessment changes in patients treated with orthognathic surgery: pre- and postsurgery report. World J Orthod 2004; 5:48-53.

14. Peiro-Guijarro MA, Guijarro-Martınez R, Hernandez-Alfaroc F. Surgery first in orthognathic surgery: A systematic review of the literature. Am J Orthod Dentofacial Orthop 2016; 149:448-62

15. Pache^co-Pereiraa C, Abreub LG, Dickc BD, Luca Cantod GD, Paivae SM, MirfAngle CF. Patient satisfaction after orthodontic treatment combined with orthognathic surgery: A systematic review Orthodontist 2016, 86 (3): 495-508.

16. Athanasiou AE, Melsen B, Eriksen J. Concerns, motivation, and experience of rthognathic surgery patients: a retrospective study of 152 patients. Int J Adult Orthod Orthognath Surg 1989; 4:47-55.

17. Chen B, Zhang ZK, Wang X. Factors influencing postoperative satisfaction of orthognathic surgery patients. Int J Adult Orthod Orthognath Surg 2002; 17:217-22.

18. Cunningham SJ, Crean SJ, Hunt NP, Harris M. Preparation, perceptions, and problems: a long-term follow-up study of orthognathic surgery. Int J Adult Orthod Orthognath Surg 1996; 11:41-7.

19. Derwent SK, Hunt NP, Cunningham SJ. A comparison of parents' and patients' views of orthognathic treatment. Int J Adult Orthod Orthognath Surg 2001; 16:171-8.

20. Finlay PM, Atkinson JM, Moos KF. Orthognathic surgery: patient expectations; psychological profile and satisfaction with outcome.Br J Oral Maxillofac Surg 1995; 33:9-14.

21. Garvill J, Garvill H, Kahnberg KE, Lundgren S. Psychological factors in orthognathic surgery. J Craniomaxillofac Surg 1992; 20: 28-33.

22. SANA TAHIR, SADAF RAFFI, IBRAHIM MW, KHAN MZ. Esthetics, functional, and psychosocial satisfaction Sep2015. Pakistan Oral \& Dental Journal; 35 (3): 395-400

23. Al-Riyami S, Moles DR, Cunningham SJ. Orthognathic treatment and temporomandibular disorders: A systematic review. Part 1 . A new quality-assessment technique and analysis of study characteristics and classifications 2009.Am J Orthod Dentofacial Orthop 2009; 136 (5): 624-5.

24. Bermell-Baviera A, Bellot-Arci's C, Montiel-Company JM, Almerich-Silla JM: Effects of mandibular advancement surgery on the temporomandibular joint and muscular and articular adaptive changes-a systematic review. Int J Oral Maxillofac Surg. 2016; 45: 545-552.

25. Kaklamanos EG, Kolokitha OE. Relation between soft tissue and skeletal changes after mandibular setback surgery: A systematic review and meta-analysis. J Cranio-Maxillo-Facial Surg 2016. 44: 427- 435

26. Co-Pereiraa CP; Abreub LG; Dickc BD; Cantod GD; Paivae SM; Mirf CF. Patient satisfaction after orthodontic treatment combined with orthognathic surgery: A systematic review 2016. Angle Orthodontist, Vol 86 (3): 495 508 . 